\title{
Los cambios en la estructura del empleo en España a lo largo del ciclo económico: patrones de cambio y factores explicativos (1995-2014)
}

\author{
Sergio Torrejón Pérez \\ Joint Research Centre / European Commission \\ sergio.torrejon-perez@ec.europa.eu \\ ORCID: 0000-0001-8623-856X
}

Recepción: 28-03-2018

Aceptación: 19-10-2018

Publicación: 30-05-2019

\section{Resumen}

En este artículo se analizan los patrones de cambio estructural del empleo a lo largo del último ciclo económico en España (1995-2014). Primero se realiza un diagnóstico en cada etapa: de cierta polarización en la fase expansiva y de mejora en la crisis. Luego se exploran las principales causas que explican esas dinámicas. Se lleva a cabo con datos de la EU-LFS y la EWCS, que se complementan con el uso de unos índices de tareas que permiten enriquecer los diagnósticos. Al seguir un procedimiento similar que muchos trabajos de referencia y clasificar los empleos con un indicador relativamente novedoso, se ofrece un diagnóstico alternativo de los cambios recientes en el empleo, lo que sirve para enriquecer el debate sobre algunas cuestiones centrales de los campos de la economía y la estratificación social.

Palabras clave: empleo; cambio estructural; polarización; ciclo económico; mercado de trabajo

Abstract. Changes in the employment structure in Spain along the economic cycle: Patterns of change and drivers (1995-2014)

This paper analyzes the patterns of structural change in employment along the last economic cycle in Spain (1995-2014). First, a diagnosis is performed for every stage of the cycle, finding polarization in the expansive period and upgrading in the crisis. The main causes that explain these dynamics are then explored. To do so, data from the EU-LFS and the EWCS are used and complemented with a set of task indices that enrich the diagnosis. Following a similar methodology to that used in the literature but classifying the different jobs with a new index, an alternative diagnosis of changes in employment is provided. This can be useful to enrich the debate on some key questions in the economics and social stratification fields.

Keywords: employment; structural change; polarization; economic cycle; labor market 


\section{Sumario}
1. Introducción
4. Análisis
2. Debate: los factores que explican los
5. Conclusiones
cambios en el empleo
Referencias bibliográficas
3. Metodología
Anexo

\section{Introducción}

La literatura sobre el cambio estructural del empleo se ha nutrido en los últimos años de unas innovaciones en el enfoque y los métodos que han contribuido a la proliferación de trabajos y la consolidación del tema como un asunto de central importancia, tanto en el campo de la economía como de la estratificación social. El caso de España también se ha tratado, aunque los trabajos son más escasos y en la mayoría de ellos la cuestión no se ha abordado en profundidad, sino como parte de estudios comparativos más amplios en los que se considera junto a los casos de otros países (Eurofound, 2008, 2011, 2013, 2014, 2015; Fernández-Macías, 2010, 2012; Goos et al., 2009, 2010, 2014; OECD, 2001, 2003; Oesch y Rodríguez Menés, 2011).

No obstante, conviene estudiar de forma detallada los casos de países concretos. Y es que si bien algunos de los factores que impulsan cambios en la demanda de empleo tienen un desarrollo e impacto similar en todos los países desarrollados - como la tecnología y los cambios en el comercio internacional-, existen otros que generan dinámicas particulares, por lo que se puede esperar que generen eventuales diferencias en los patrones de cambio estructural en cada lugar (Eurofound, 2013: 25-26; Fernández-Macías, 2012: 2; Oesch y Rodríguez Menés, 2011: 505).

El caso de España es interesante porque la evolución reciente del ciclo económico ha evidenciado la extrema sensibilidad del empleo a los cambios en la demanda productiva (CES, 2012: 386; García Serrano, 2011: 162163; Garrido, 2010: 49; Torrejón, 2016, 2017a). Un problema que tiene implicaciones y está relacionado con los cambios en la estructura de empleo. No obstante, se ha demostrado que España es el caso más cíclico de entre varios países del entorno, con unos patrones de cambio del empleo que muestran diferencias muy marcadas entre lo que ocurre en fases expansivas y de crisis (Eurofound, 2015: 46-47). Por ello, se espera que la volatilidad del empleo afecte tanto a la intensidad de los flujos como a los cambios en su estructura.

El propósito de este artículo es llenar el vacío que existe en cuerpo de la investigación sobre el cambio estructural del empleo en España, abordando la dinámica y particularidades del caso en profundidad y con información que me permite analizar lo ocurrido a lo largo del ciclo económico completo - durante la fase expansiva y de crisis-. Este interés se materializa con las siguientes preguntas de investigación: ¡cuáles son los patrones de cambio en 
la estructura de empleo en cada fase del ciclo?, ¿qué factores y cambios están relacionados con las particularidades del caso de España?

Para garantizar cierta claridad, el contenido se expone del siguiente modo: en primer lugar, se hace un repaso de los argumentos de la literatura internacional sobre el cambio estructural del empleo. El propósito es recapitular para orientar y enmarcar los resultados que se obtienen en el mismo. En segundo lugar, se escribe sobre los métodos empleados. Los dos siguientes apartados conforman el núcleo analítico del trabajo: en el primero, se lleva a cabo un diagnóstico de los patrones de cambio estructural que hemos experimentado en España en cada fase del ciclo económico; y en el segundo, se exploran cuáles son los factores y las causas relacionados. Finalmente se remarcan las aportaciones principales del trabajo.

\section{Debate: los factores que explican los cambios en el empleo}

\subsection{Un problema multicausal: el cambio tecnológico como proceso más influyente}

Daniel Bell anunció a principios de los setenta que la introducción de innovaciones científicas y técnicas en la producción iba a hacer que aumentaran las ocupaciones de carácter profesional y técnico, mientras que la automatización provocaría una reducción del peso de las ocupaciones tradicionales de cuello azul de la industria (1973). Esto produciría un cambio progresivo de la industria a los servicios, generando un proceso de mejora ocupacional. Esta fue una de las primeras grandes obras que llevaron a cabo una lectura optimista de los cambios que producía la introducción de las tecnologías de la información y la comunicación (TIC) en la organización de la producción, pero no sería la última.

La SBTC (Skill-Biased Technological Change) recogió el testigo, haciendo uso de un modelo dicotómico del mercado laboral para sostener que la introducción de las TIC genera un incremento de la demanda de mano de obra cualificada con relación a la no cualificada (Fernández-Macías, 2012: 3; Oesch y Rodríguez Menés, 2011: 506). La evidencia de algunos estudios, basados en los casos de Europa occidental y los EE. UU. (Bekman et al., 1998; Gallie et al., 1998), hizo que a finales de los noventa se generara consenso sobre la existencia de un proceso de mejora ocupacional.

Algunos analistas no quedaron satisfechos con la simplicidad de este modelo, por lo que elaboraron otros más complejos. El más conocido es el de Autor et al. (2003), que permite clasificar los empleos en función del tipo de tareas que desarrollan en el proceso productivo. Para distinguir las tareas, emplearon dos ejes: el primero distingue las tareas rutinarias de las no rutinarias; y el segundo, las manuales de las intelectuales o cognitivas. Haciendo uso de esta clasificación, plantearon la RBTC (Routine-Biased Technological Change), que explica que las nuevas tecnologías son complementarias e impulsan las tareas no rutinarias que exigen flexibilidad, creatividad, capacidad de resolución de problemas y otros atributos similares, mientras que pueden sustituir 
fácilmente tareas rutinarias y que pueden describirse con reglas programables (Autor, 2014: 846; Autor et al., 2003: 1.322). Desde entonces, muchos trabajos se han basado en el mismo modelo, lo que permitió completar la panorámica: según la RBTC, la tecnología puede remplazar fácilmente tareas rutinarias - localizadas en empleos de calidad intermedia - y es complementaria y favorece las no rutinarias - tanto manuales como cognitivas, localizadas en ambos extremos de la distribución-, por lo que en consecuencia sus previsiones se volvieron más pesimistas.

Por ejemplo, Goos y Manning concluyeron en un análisis del Reino Unido que desde los setenta, debido al desarrollo de las TIC, fueron los mejores y peores empleos los que se expandieron con relación a los de calidad intermedia (2007). Tras este, muchos trabajos han revisado el modelo original y concluido que el desarrollo de las TIC ha tenido un efecto polarizador sobre las estructuras de empleo no solo del Reino Unido, sino también de los EE. UU. (Acemoglu y Autor, 2011; Autor y Dorn, 2013; Autor et al., 2013; Autor et al., 2006; Wright y Dwyer, 2003) y la mayor parte de países europeos (Acemoglu y Autor, 2011; Goos et al., 2009, 2010, 2014). La evidencia que ha mostrado la regularidad de ese patrón en los EE. UU. y algunos países europeos ha hecho que la tesis de la polarización se generalizara y extrapolara a la experiencia de la mayor parte de países desarrollados (Fernández-Macías, 2012), y ha adquirido una influencia notable también en España, donde se ha asumido como cierta (Muñoz, 2014: 355) o se ha generado evidencia que apunta en ese sentido (Bernardi y Garrido, 2008: 303-304).

Aunque difieran en las previsiones que hacen, los argumentos de la SBTC y la RBTC tienen algo en común: la atención casi exclusiva al fenómeno del cambio tecnológico. Estos cambios tienen un desarrollo similar en los países desarrollados, motivo por el que se espera que tengan también un impacto similar. Esto explica que muchos analistas llevaran a cabo diagnósticos similares para los casos de muchos países del entorno.

En cambio, estas aproximaciones contrastan con las de quienes han explorado la influencia de otros factores que dan lugar a procesos distintos en cada lugar. La configuración institucional o la composición de la oferta de trabajo tienen características particulares en cada país, por lo que se puede esperar que sesguen el patrón homogéneo al que inducen los factores de demanda, dando lugar a una diversidad de patrones en el escenario internacional (Eurofound, 2008, 2011, 2013, 2014; Fernández-Macías, 2012; Oesch y Rodríguez Menés, 2011). Hay, por ejemplo, quien ha analizado la influencia de los modelos de estados de bienestar, canalizando a través de un tipo de empleo u otro el crecimiento de la demanda de trabajo (Esping-Andersen, 1999). Pero las diferencias no se limitan a las establecidas por modelos en los que se enmarcan conjuntos de países, sino que en cada uno intervienen otra serie de factores institucionales, de oferta y sociodemográficos que conforman la singularidad de los mercados de trabajo nacionales. Incluso debe tenerse en cuenta la influencia que pueden tener, en un nivel más micro, las relaciones de poder y otros factores sociales, como reveló Braverman al argumentar que la carga de rutina asociada 
a un trabajo (sirviéndose del modelo industrial fordista como ejemplo) también sirve como herramienta para reducir el grado de control sobre el proceso de trabajo de los trabajadores (1974). Considerar todas estas aportaciones sirve, en definitiva, para asumir la naturaleza multicausal de los problemas que se abordan. Algo que evidencia una gran complejidad, pero que se debe tener en cuenta para llevar a cabo análisis de caso en profundidad con los que obtener diagnósticos más ajustados y realistas.

La motivación principal de este trabajo es, en definitiva, hacer un diagnóstico del cambio estructural que recoja la influencia de, al menos, los factores más importantes que generan cambios en el empleo. Es por eso que, para tratar de identificarlos, los patrones de cambio estructural se desagregan usando algunas variables sociodemográficas básicas, como se detalla luego en el apartado de análisis. ${ }^{1}$

\subsection{Los cambios en la estructura de empleo en España}

- Dado que interesa profundizar sobre las características del cambio estructural del empleo en España, se exponen a continuación los resultados de algunos de los principales estudios realizados previamente que trataron este caso. Esto permitirá contrastar los resultados de este análisis con los de algunos predecesores. Sobre lo que ocurrió durante la expansión no hay acuerdo. Muchos estudios comparten un diagnóstico optimista, y hablan de un proceso de mejora del empleo con un destacado crecimiento de los empleos de calidad intermedia - sobre todo con relación al menor crecimiento relativo de los de la parte baja de la distribución (Eurofound, 2008: 12, 2013: 30, 2014: 36; Fernández-Macías, 2012: 15)—. España no solo formaría parte del conjunto de países que han experimentado ese patrón, sino que se ha llegado a considerar como ejemplo paradigmático (Eurofound, 2008: 14). Otros trabajos aportan datos aún más positivos y hablan sin matices de un proceso de mejora del empleo, considerando que los empleos mejor remunerados crecieron más que los peor pagados (OECD, 2001: 111, 2003: 41; Oesch y Rodríguez Menés, 2011: 514). Sin embargo, también existen análisis que sugieren que España forma parte del grupo de países en que se polarizó la estructura de empleo (Bernardi y Garrido, 2008; Goos et al., 2009: 61, 2010: 48, 2014: 5; Martín y Gibert, 2006).

- En los escasos trabajos que han tratado lo ocurrido en España durante la crisis, se observa un mismo resultado: un proceso de polarización al que parece que han convergido la mayor parte de países (Eurofound, 2013: 30)

1. El propósito no es establecer mecanismos causales que determinen el peso de cada uno de los posibles efectos intervinientes en los procesos de cambio estructural. La complejidad de un fenómeno multicausal como este y las limitaciones de las herramientas y las bases de datos que se utilizan impiden abordar tal tarea. Lo que se pretende, en cambio, es realizar un examen exploratorio que arroje luz sobre la vinculación entre los principales cambios de demanda, oferta e institucionales y los cambios en el empleo. 
y que se pronosticaba que ocurriría en España (Muñoz de Bustillo y Antón, 2011). Otros trabajos posteriores consideran que ha ocurrido esto tanto de 2008 a 2014 (CaixaBank, 2015: 28) como en la primera y segunda fase de crisis —de 2008 a 2010 (Eurofound, 2011: 19, 2013: 30, 2014: 36), y de 2011 en adelante (Eurofound, 2014: 36, 2015: 15)—. Este acuerdo puede deberse a que la variedad de análisis y estrategias metodológicas es todavía menor o a un hecho contrastado en la literatura sobre ciclos económicos: que las etapas de crisis son etapas de cambio estructural más intenso que las expansivas (Perez, 1983).

\subsection{El enfoque del jobs approach}

El objeto de estudio de este trabajo aborda algunas de las temáticas más prolíficas en economía y sociología, por lo que el apartado de debate podría prolongarse fácilmente. Sería interesante incorporar explicaciones distintas, como las de quienes proporcionan información relevante sobre el marco institucional (Crouch, 1994; Gallie, 2007; Gallie et al., 1998; Hall y Soskice, 2001) o las de quienes, centrándose en el caso español, han trabajado la cuestión de la calidad del empleo desde un enfoque distinto, prestando más atención a los conflictos en las relaciones laborales y el marco normativo (Arnal et al., 2012; Castillo, 2010; Miguelez y Prieto, 2009).

Sin embargo, dado que conviene acotar la discusión, este trabajo se centra sobre todo en la parte de la literatura que se aproxima al objeto de estudio a través del jobs approach (o marcos similares): un enfoque que hace referencia a la unidad de análisis de referencia (los empleos) y a la forma en que se analiza el cambio estructural (mostrando la evolución de la ocupación en grupos de empleos semejantes, clasificados según su nivel de calidad u otra característica relacionada). ${ }^{2}$

Esto es relevante porque el uso de este enfoque tiene numerosas implicaciones, y algunas de ellas ofrecen ventajas claras. Primero que, como veremos luego, la unidad de análisis (producto de la combinación de ocupaciones y sectores) ofrece una definición precisa e intuitiva de los empleos: los sectores hablan del valor económico y social que se produce, y las ocupaciones de la forma en que se produce. La combinación de ambas genera, por tanto, categorías más detalladas y precisas, lo que resulta de gran utilidad en términos teóricos. En segundo lugar, estas categorías también son útiles en términos analíticos, ya que disponemos de clasificaciones internacionales homologadas para clasificar tanto los sectores (la Nomenclatura Estadística de Actividades Económicas de la Comunidad Europea, NACE) como las ocupaciones (Clasificación Internacional Uniforme de Ocupaciones, CIUO). Además, por estas y otras razones se trata de uno de los enfoques más utilizados desde la década de los noventa hasta la actualidad, por lo que su adopción permite aportar y

2. En el apartado sobre la metodología se explican los detalles acerca del procedimiento y el enfoque. 
establecer un diálogo directo con parte de la literatura más relevante sobre el objeto de estudio tratado.

\section{Metodología}

Como se relataba, para cumplir con los objetivos propuestos y entablar un diálogo con la literatura de referencia (discutida en el apartado anterior) se utiliza el mismo procedimiento que utilizan muchos de esos trabajos. Consta de dos pasos básicos:

1. Clasificar los empleos en grupos de un tamaño similar según su nivel de calidad.

2. Ver dónde se ha concentrado el aumento de la ocupación en diferentes etapas.

Sin embargo, se incorpora una novedad con la que se complementa la información y que trata de aportar valor añadido, y que se explica luego con mayor detalle. Primero conviene aclarar qué son los empleos según el jobs approach: el producto de la combinación de ocupaciones y sectores, detallados al nivel de dos dígitos de acuerdo con las clasificaciones CIUO y NACE. Una ocupación, por ejemplo, son los trabajadores de servicios personales. Un sector común, el de la educación. Teniendo en cuenta esto, uno de los muchos empleos que existen es el de los trabajadores de servicios personales en el sector de la educación. Otro, los trabajadores de servicios personales en el sector de las actividades de salud humana, y así sucesivamente con todas las posibles combinaciones de ocupaciones y sectores.

La cantidad de empleos varía de un periodo a otro, debido a los cambios que ha habido en las clasificaciones de sectores y ocupaciones. Las rupturas habidas en la European Union Labour Force Survey (EU-LFS) a lo largo de las distintas olas de la encuesta dan lugar a combinaciones diferentes en tres subperiodos: de 1995 a 2007 se combina CIUO88 y NACE1.1, lo que da lugar a 1144 empleos; de 2008TI a 2010TIV se combina CIUO88 y NACE2, lo que da lugar a 1427 empleos; finalmente, desde el primer trimestre de 2011 se utiliza CIUO08 y NACE2, de cuya combinación se extraen 1955 empleos.

Los trabajos de Fina (1984) y Garrido y Toharia (1991) fueron precursores en el uso de este enfoque en España. Pero quien lo dio a conocer a nivel internacional fue Stiglitz, que lo aplicó para un análisis del mercado laboral estadounidense en un informe del Council of Economic Advisers (1996). El procedimiento fue perfeccionado luego en trabajos como el de Wright y Dwyer (2003), y su uso extendido en la década siguiente al análisis de las experiencias de países europeos a finales del siglo XX y principios del siglo XXI (Eurofound, 2008, 2011, 2013, 2014, 2016; Fernández-Macías, 2010; Fernández-Macías et al., 2012; Goos y Manning, 2007; Goos et al., 2009).

En la mayor parte de trabajos que utilizan este enfoque — como hacen quienes estudian el cambio ocupacional (Acemoglu y Autor, 2011; Garrido, 
2008a; Garrido y Rodríguez, 2011; Goos et al., 2009, 2014; Oesch y Rodríguez Menés, 2011)—, se utilizan proxis para medir la calidad de los empleos y poderlos clasificar en grupos de un tamaño similar. Los más habituales se basan en información sobre los ingresos o el nivel educativo, ya que se trata de indicadores que correlacionan mucho con el nivel de calidad del empleo. A cada uno de ellos se le asigna un valor medio a través del uso de estos indicadores, lo que permite clasificarlos de forma jerárquica. El procedimiento de este trabajo sigue la misma lógica, aunque se incorpora aquí una primera novedad. En vez de utilizar estas variables para asignar una medida de calidad, se usa un indicador multidimensional: el Indice no Pecuniario de Calidad del Empleo (NPI). Su valor equivale a la media de varios de los componentes que determinan la calidad del empleo ${ }^{3}$. La construcción de este índice se ha basado en los trabajos de Muñoz de Bustillo et al. (2011), Eurofound (2013: 43-51) y Fernández-Macías et al. (2014), donde explican cuáles son las variables a partir de las que se construyen los ítems de que se compone, el peso que se otorga a cada uno y el resto de detalles sobre la operacionalización. La base de datos de donde se extrae la información es la Encuesta Europea sobre las Condiciones de Trabajo (EWCS). En concreto las olas de 1991, 1995, 2000, 2001, 2005 y 2010. El valor final del índice equivale a una media de la calidad de los empleos a lo largo del periodo considerado.

$\mathrm{Al}$ proceder como han hecho tantos trabajos y emplear un indicador distinto, se ofrece evidencia alternativa sobre el cambio estructural del empleo, ya que esta información no ha sido utilizada con este propósito en el caso de España. Pero la innovación no es un fin en sí misma, sino que considero que su uso aporta algunas ventajas. El NPI es un índice multidimensional, más completo y consistente. Equivale a la media de varios indicadores, por lo que se reduce la probabilidad de obtener una información parcial y sesgada, como puede ocurrir al utilizar indicadores individuales. Un ejemplo real que ayuda a entender este riesgo es el de España en la etapa expansiva. En este periodo los ingresos de sectores relacionados con la burbuja crecieron mucho, mientras que el resto de condiciones de trabajo permanecieron inalteradas. Considerando esto, puede resultar problemático utilizar los ingresos como indicador de calidad, ya que puede que no esté midiendo lo que se pretende, sino que refleje el propio proceso de burbuja. Al exponer mis resultados, profundizaré sobre la naturaleza del problema y sus implicaciones a la hora de llevar a cabo un diagnóstico.

El siguiente paso, tras otorgar un valor de calidad a los empleos (para lo que se usa el NPI), es agruparlos en quintiles: cinco grupos del mismo tamaño, con los de menor calidad en un extremo - a la izquierda en los gráficos- y

3. El NPI mide la calidad del empleo utilizando los siguientes componentes y subcomponentes: la calidad intrínseca del trabajo (habilidades requeridas, grado de autonomía, nivel de soporte social); la calidad del empleo (estabilidad contractual, oportunidades de desarrollo); los riesgos del lugar de trabajo (riesgos físicos, riesgos psicosociales), y la jornada de trabajo y conciliación (duración, programación, flexibilidad e intensidad de la misma). En los trabajos citados, se explicitan las variables de la EWCS que se utilizan para medir esos subcomponentes y elaborar los componentes generales y el índice. 
los de mayor calidad en el otro. Lo que se hace en el análisis es desplegar estos quintiles en diagramas que reflejan el cambio del número de ocupados en cada grupo del inicio al final de cada periodo. De este modo se determina en qué tipo de empleos se ha concentrado el crecimiento, y se obtiene un diagnóstico del cambio estructural en cada una de las etapas del ciclo económico. Esto nos permite conocer las implicaciones de los cambios en el empleo desde el punto de vista de la desigualdad, tarea a la que se dedica la primera parte del análisis.

Finalmente, la EU-LFS permite combinar la información sobre los empleos con algunas variables sociodemográficas y del mercado de trabajo, por lo que se desagregan esos patrones para profundizar acerca de las fuerzas que impulsan los cambios descritos — segunda parte del análisis-. Entre los principales factores, se incluyen la recepción de grandes flujos de inmigrantes, el impacto de la burbuja inmobiliaria en la estructura de empleo o la dinámica del empleo femenino. ${ }^{4}$

Una vez resueltas las cuestiones centrales sobre el método, se debe advertir que se añade información complementaria que no afecta al diagnóstico de cambio estructural, pero sirve para enriquecerlo y obtener una imagen detallada de los cambios descritos. Para ello se utilizan los índices de tareas elaborados para el proyecto European Jobs Monitor -EJM- (Fernández-Macías et al., 2016). Al vincular la información de estos índices a los empleos de los diagramas, podemos hablar no solo de si los que más crecen son los de calidad baja o alta, sino también de si se trata de los más o menos rutinarios, físicos, intelectuales, dotados de mayor o menor autonomía, etcétera. Es decir, se trata de una herramienta que sirve para calificar los empleos y la naturaleza del cambio estructural. Esto, además, permite contrastar hipótesis que ponen en relación la dinámica del empleo y sus características materiales. Una línea de investigación que está generando mucho interés y a la que buenamente se puede contribuir con una información tan detallada. En las tablas del anexo muestro los índices usados y la puntuación de cada grupo de empleos, por lo que ofrecen toda la información necesaria para interpretar los gráficos del análisis.

\section{Análisis}

\subsection{Los patrones de cambio estructural a lo largo del ciclo económico}

La fuerte elasticidad del empleo a los cambios en la producción distingue al mercado de trabajo español (Torrejón, 2016, 2017a). Según la Encuesta de

4. Es imposible abarcar en un estudio todos los factores que median en cualquier fenómeno social. Sin embargo, las bases de datos utilizadas permite desagregar los análisis con algunas variables sociodemográficas básicas de la EU-LFS, lo que permite arrojar luz sobre la relación entre los cambios observados y algunos de los principales cambios sociales y económicos vividos en las últimas décadas. Sería interesante incorporar otras variables complementarias, aunque por el momento, al haber tenido que combinar muchas bases de datos para generar la matriz de este estudio (los datos sobre el empleo con los indicadores de tareas), esta permite contar con un número limitado de variables sociodemográficas. 
Población Activa, en la última etapa expansiva el número de ocupados aumentó en más de 8 millones, y pasó de poco más de 12 en el primer trimestre de 1994 a casi $21-20.753 .400$ - en el tercer trimestre de 2007. A partir de esa fecha, la crisis hizo que ese logro se desdibujara, al menos parcialmente, ya que en seis años y medio el número de ocupados se redujo en casi 4 millones - 3.802.800- Un momento, aquel primer trimestre de 2014, en el que la tendencia de la ocupación a la baja se detuvo y alcanzó un nuevo punto de inflexión.

A pesar de lo sensible que es en España el empleo a los cambios en la producción, no existe una relación clara entre el ciclo económico y las cuestiones relacionadas con el bienestar social y la desigualdad. El fuerte incremento del número de ocupados en la etapa expansiva creó un clima de optimismo con la situación económica, lo que provocó que se hablara de ella con términos triunfalistas. Pero este crecimiento, para sorpresa de muchos, no se tradujo en una mejora significativa de los indicadores de desigualdad y pobreza (Ayala, 2014: 43). Esto pone de manifiesto la difusa y compleja relación entre la dinámica del ciclo económico y la desigualdad y la pobreza (Carabaña, 2016; Carabaña y Salido, 2010). Y es que los cambios en el volumen del empleo no son lo único que importa, sino que hay que considerar las características del que se crea para entender los fenómenos relacionados con estas cuestiones.

Esta es la razón por la que conviene analizar la relación entre el ciclo económico y los cambios en la estructura de empleo. Para llevarlo a cabo primero se examina, como hicieron Wright y Dwyer en el caso de los Estados Unidos (2003), si el crecimiento del empleo en la expansión en España contribuyó a un proceso de mejora de su estructura o hizo que se polarizara. Además, ya que disponemos de datos para hacerlo, también me fijaré en lo que ha ocurrido luego durante la crisis.

El primer gráfico revela que el patrón de cambio estructural en la etapa expansiva se asemeja a uno de polarización. Aunque no responde al tipo ideal

Gráfico 1. Evolución del $n^{\circ}$ de ocupados (miles) en quintiles, 1995-2007

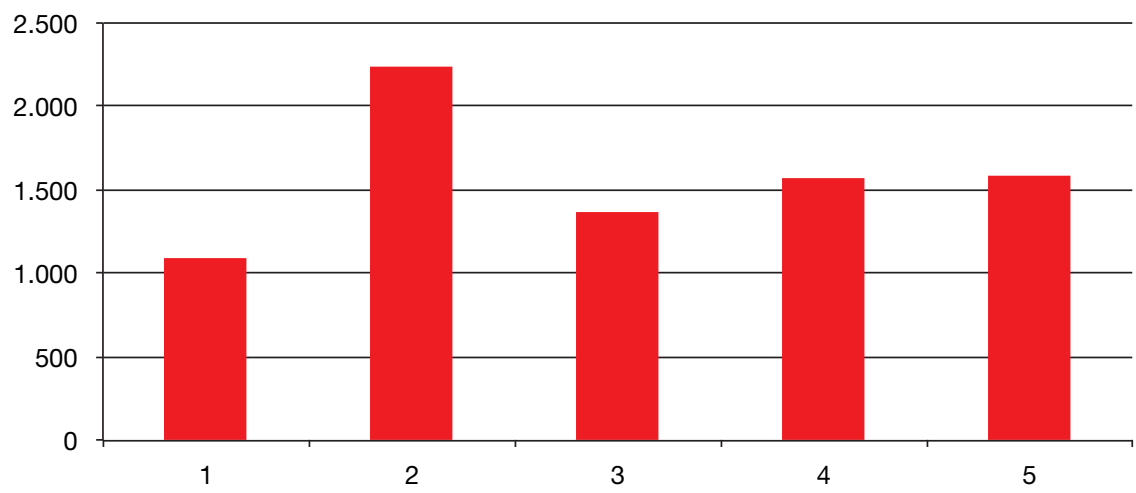

Fuente: elaboración propia a partir de la EU-LFS y la EWCS. 
de modelo de bipolarización con los extremos dilatados con relación al centro, sino que se aprecian matices. En general, los empleos que más crecieron fueron los de calidad intermedia baja - sobre todo del segundo quintil, nutrido de empleos muy físicos y de carácter rutinario-y los de mayor calidad — de los dos quintiles superiores, donde se ubican los empleos con un fuerte carácter intelectual, que exigen mucha interacción social, están dotados de mayor autonomía e implican un uso intensivo de las TIC.

Este diagnóstico se aproxima a los que concluyen que en España, durante la fase alcista, los empleos menos dinámicos fueron los de calidad intermedia (Bernardi y Garrido, 2008; Goos et al., 2009, 2010, 2014; Martín y Gibert, 2006). En cambio, contrasta con el de quienes han hecho una lectura optimista sobre lo ocurrido en esta etapa (Eurofound, 2008: 12, 2013: 30, 2014: 36; Fernández-Macías, 2012: 15; OECD, 2001: 111, 2003: 41; Oesch y Rodríguez Menés, 2011: 514). Esta discrepancia se debe, fundamentalmente, a algo que señalaba en el apartado de metodología: el uso de un indicador de calidad distinto.

Muchos de los análisis que hablan de que durante estos años hubo un proceso de mejora del empleo con un importante crecimiento de los de calidad intermedia (mid-upgrading) tienen algo en común: el uso de proxis basados en la información sobre los salarios. Al centrarse en esta única dimensión, los resultados no solo reflejan la calidad del empleo, sino también una de las consecuencias del propio proceso de burbuja, que hizo que los salarios de empleos de baja productividad vinculados a la misma se inflaran y se desplazaran de la base de la estructura a posiciones intermedias. Lo más relevante es que fueron precisamente estos algunos de los empleos que más crecieron en la época, por lo que al proceder de este modo se puede sesgar el diagnóstico que se obtiene sobre el cambio estructural, lo que hace que se sobreestime el crecimiento de los de calidad intermedia e infraestime el de los de menor calidad. ${ }^{5}$

Los gráficos 2 y 3 muestran que, tras la fase alcista, la destrucción de empleo asociada a la crisis generó una dinámica de cambio estructural radicalmente diferente. Tanto en la primera fase de crisis como en la segunda, el patrón se ajusta a uno de mejora ${ }^{6}$, siendo los peores empleos - los más físicos, rutinarios y que exigen un uso más intensivo de maquinaria mecánica- los que han sufrido más intensamente el ajuste. El contraste lo ofrecen los empleos de mayor calidad —en los que se desarrollan en mayor medida tareas de carácter intelectual, que exigen mucha interacción social, dotadas de mucha autonomía

5. No obstante, diferenciar los empleos por niveles salariales tiene interés, puesto que son la principal fuente de renta de los individuos y reflejan otras dinámicas sociales. Conviene hablar, más que de indicadores mejores o peores, de indicadores distintos y complementarios.

6. Es preciso aclarar que se habla proceso de mejora (upgrading) cuando el saldo total de la ocupación, en un periodo determinado, es favorable para los empleos de más calidad. Es decir, cuando en un periodo la proporción de ocupados en empleos de calidad aumenta. Esto no quita que tal proceso, que afecta solo al universo de los ocupados (no se considera, por tanto, a la gente inactiva o parada) pueda tener enormes costes sociales y se haya podido producir a costa de una enorme destrucción del empleo, como es el caso. 
Gráfico 2. Evolución del nº de ocupados (miles) en quintiles, 2008TI-2010TIV

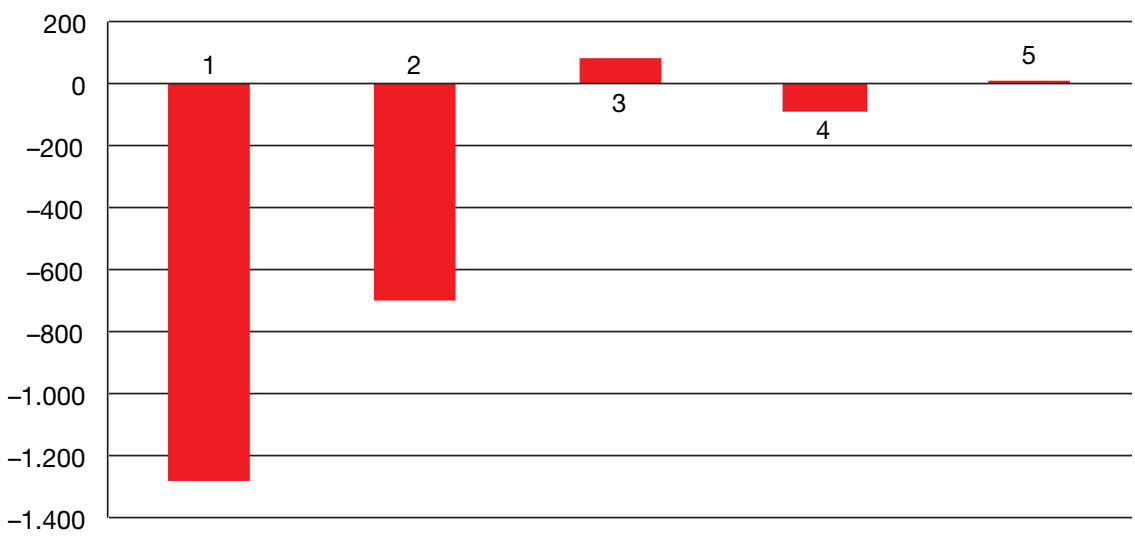

Fuente: elaboración propia a partir de la EU-LFS y la EWCS.

Gráfico 3. Evolución del nº de ocupados (miles) en quintiles, 2011TI-2014TII

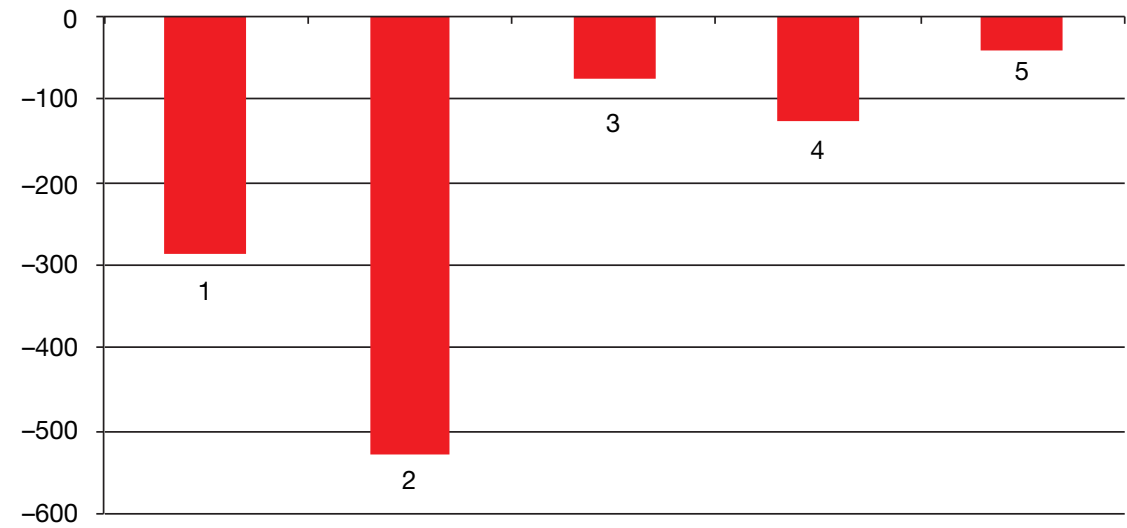

Fuente: elaboración propia a partir de la EU-LFS y la EWCS.

y que exigen un uso intensivo de herramientas relacionadas con las TIC-, que han resistido mejor la caída de la demanda.

La principal diferencia entre lo ocurrido en una primera y en una segunda fase de crisis es que en el primer caso se observa un patrón de mejora nítido: casi escalonado, con un ajuste centrado en los empleos del primer quintil y que va teniendo un impacto menor a medida que aumenta el nivel de calidad de los empleos. En la segunda fase de crisis, la caída de la ocupación se extendió y empezó a afectar más a empleos de mayor calidad. Esto se debe, en gran medida, al efecto de la caída de la demanda agregada asociado a toda crisis. 
Aunque en un primer momento la crisis afecte de manera más virulenta a los ocupados en sectores de actividad en que se hunde la demanda, la caída del poder adquisitivo de estos trabajadores y la merma de su capacidad de consumo termina afectando a la demanda de otro tipo de bienes y servicios, por lo que los efectos de la crisis se transmiten y extienden de unos sectores a otros.

Volviendo al diagnóstico de cambio estructural durante la crisis, debo señalar que el que se ofrece aquí contrasta con el de los trabajos revisados en el apartado 2.2., que subrayan que durante estos años España se sumó a la lista de países que han convergido hacia dinámicas polarizadoras (Eurofound, 2011: 19, 2013: 30, 2014: 36, 2015: 15). La causa de esta discrepancia es la misma: el uso de un indicador de calidad distinto. El ajuste de la crisis se ha centrado en empleos de baja productividad de sectores relacionados con la burbuja. Al emplear un indicador basado en los ingresos - como hacen los trabajos mencionados-, estos tienden a ubicarse en posiciones intermedias, y esto hace que al caer sean los empleos de los extremos de la distribución los que con relación a estos parecen haber resistido mejor. Como se explica en el apartado de metodología, la intención de evitar este sesgo fue clave para elegir un indicador multidimensional, modo con el que creo que se puede ofrecer un diagnóstico complementario y más realista. ${ }^{7}$

\subsection{Los factores que explican las particularidades del cambio estructural en España}

Tras realizar diagnósticos sobre el cambio estructural en cada etapa del ciclo económico, se trata de arrojar luz sobre algunos de los factores que explican esos resultados. Para hacerlo se desagregan los datos de la evolución de los quintiles usando variables que permiten poner en relación la dinámica del empleo con algunas características de la oferta y la demanda de trabajo.

En los siguientes gráficos figuran los patrones desagregados por sexo. Revelan que el crecimiento de la ocupación masculina durante la expansión estuvo más disperso y concentrado en los extremos de la distribución, mientras que el de las mujeres se distribuyó de forma más equitativa y nutrió sobre todo empleos de calidad intermedia. Parece que la incorporación de la mujer al mercado laboral contribuyó a atenuar la dinámica polarizadora del empleo masculino.

$\mathrm{Y}$ es que la incorporación masiva de mujeres al trabajo remunerado no se ha llevado a cabo a través de uno o escasos canales, sino que ha sido un proceso bastante heterogéneo. Excepto en los empleos más físicos, rutinarios y de menor calidad — donde su participación es aún poco intensa—, el aumento de la participación femenina estuvo muy repartido. Esto puede explicarse por el doble efecto que ha promovido la participación de la mujer en la vida activa:

7. En un informe de Eurofound se presentan resultados con un indicador como el empleado aquí y se discuten las diferencias entre unos y otros, por lo que su lectura ayuda a cualificar el artículo (Eurofound, 2013). 
Gráfico 4. Evolución del $n^{\circ}$ de ocupados (miles) en quintiles, según sexo

1995-2007

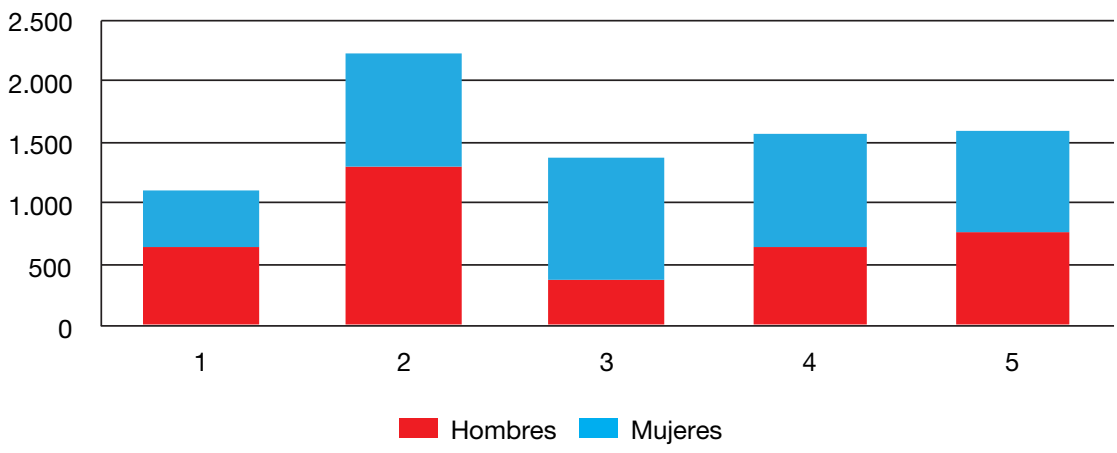

2008TI-2010TIV
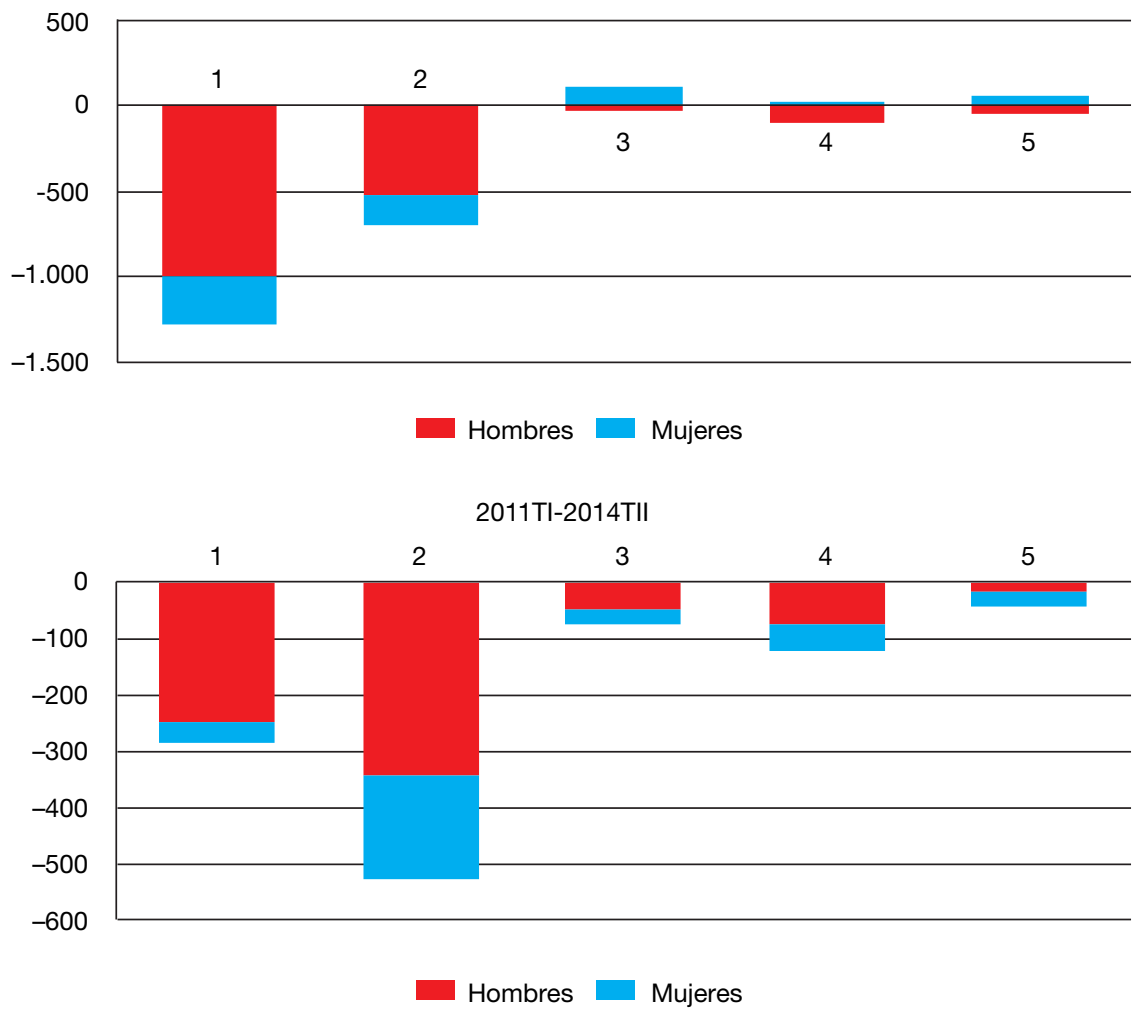

Fuente: elaboración propia a partir de la EU-LFS y la EWCS. 
- El efecto educación, que pone en relación la mayor participación laboral de las mujeres con su mayor logro educativo, y explicaría el crecimiento de su actividad en empleos de calidad intermedia-alta, enfocados a servicios en sectores como la educación o la sanidad. Este efecto es más reciente y está relacionado con la incorporación de mujeres de clases acomodadas - clase media y alta - al mercado laboral, por lo que no explica que la participación femenina haya sido tan intensiva en empleos de calidad intermedia-baja.

Prueba de ello es que, si consideramos solo a las mujeres españolas -quienes cuentan normalmente con un estatus socioeconómico más alto- vemos que de 2000 a 2008 es en las mejores ocupaciones donde más aumentó su presencia en el empleo, mientras que al incluir a las inmigrantes su participación en el empleo se diversifica y reparte (Garrido, 2008a: 40-41). Para aclarar esto, a continuación se examinan también las diferencias según nacionalidad.

- De modo que el aumento de la participación femenina en empleos de calidad media y sobre todo baja puede estar más relacionado con el conocido como efecto necesidad, que habría empujado tradicionalmente a las mujeres de clases humildes al empleo para contribuir al sustento del hogar. Este es el motivo que habría hecho que muchas mujeres se emplearan en servicios de menor calidad, como los personales y de cuidados. De hecho, hay estudios que inciden en que la contribución de las mujeres a la polarización a través de su participación intensiva en empleos de cuidados es un efecto que se ha menospreciado tradicionalmente en la literatura (Dwyer, 2013).

Estas explicaciones ofrecen un marco para interpretar las razones por las que el crecimiento de la ocupación femenina estuvo tan repartido. Algo que hizo que su incorporación a la vida activa contribuyera a atenuar un proceso de polarización.

Mientras que el crecimiento del empleo estuvo más repartido, posteriormente su destrucción se concentró en la parte baja de la distribución y ha tenido una cara predominantemente masculina — gráficos $4 \mathrm{~b}$ y $4 \mathrm{c}-$. En este caso el efecto composición por género es muy claro. La dinámica de ajuste sobre el empleo masculino de baja calidad ha contribuido de forma decisiva a acentuar el proceso de mejora de la estructura de empleo experimentado con la crisis, ya que la destrucción del empleo femenino ha sido menos intensa.

Junto a la incorporación de la mujer al mercado laboral, otro de los factores que explican el crecimiento masivo de la ocupación en las últimas décadas fue la entrada masiva de inmigrantes en nuestro país. Su proceso de inserción al mercado laboral siguió una pauta muy definida: del crecimiento total del número de ocupados en los empleos de menor calidad —en los quintiles 1 y 2 - un $64 \%$ fueron inmigrantes. Su contribución al sobredimensionamiento de la base de la estructura de empleo fue clave y su impacto sobre el resto muy escaso - gráfico 5 a.

Los flujos de inmigrantes conformaron un stock de mano de obra que se utilizó de forma generalizada para cubrir los empleos más físicos y de menor 
Gráfico 5. Evolución del $n^{\circ}$ de ocupados (miles) en quintiles, según nacionalidad

1995-2006

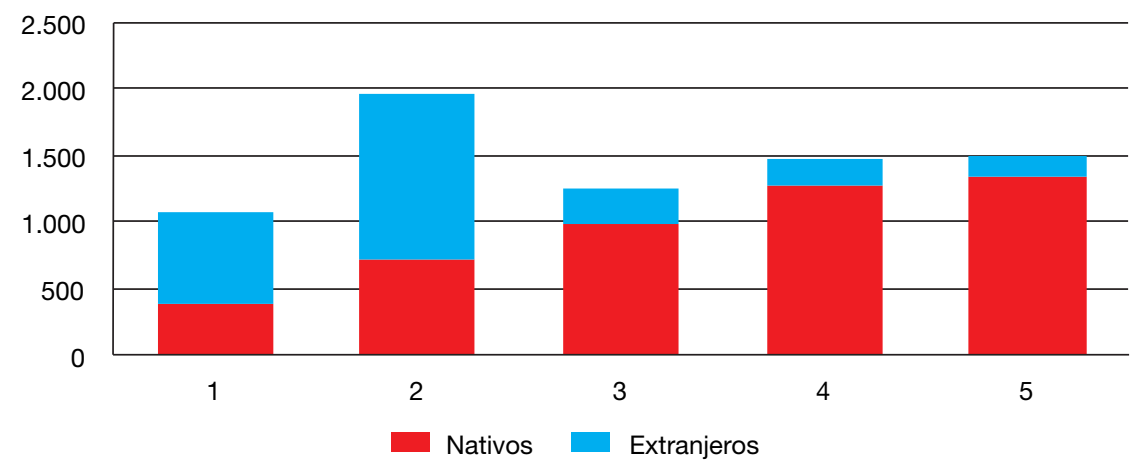

2008TI-2010TIV

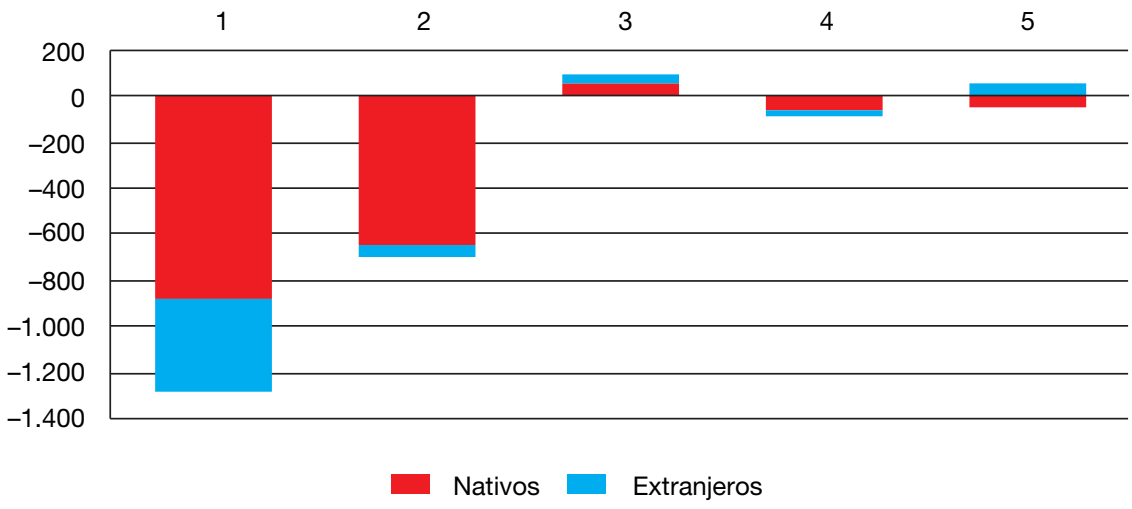

2011TI-2014TII

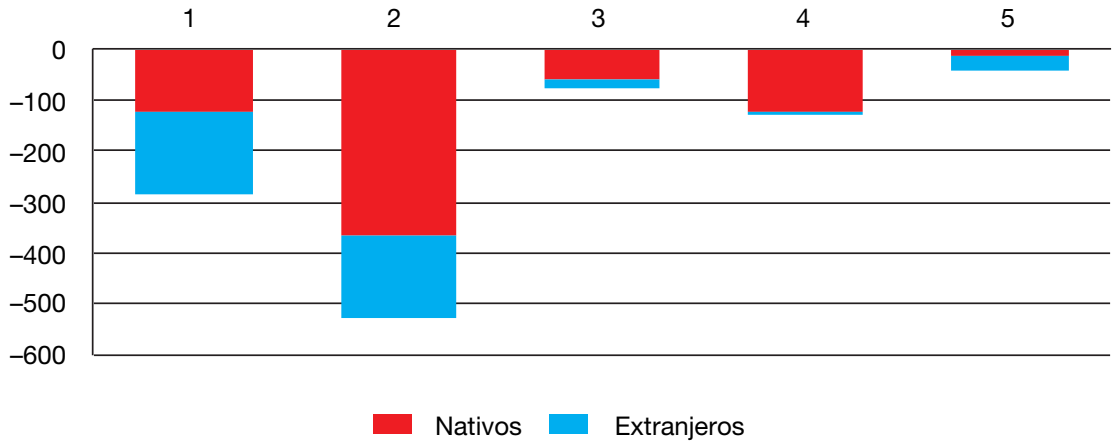

Fuente: elaboración propia a partir de la EU-LFS y la EWCS. 
calidad, con una fuerte concentración de este colectivo en los empleos de menor nivel dentro de la estructura ocupacional (Cachón, 1997; Garrido, 2008b). Prueba de ello es que del aumento total de la ocupación extranjera, un $75,3 \%$ se localizó en empleos de los dos primeros quintiles. En comparación, fueron residuales los casos en que se incorporaron a la vida activa a través de ocupaciones y sectores de mayor calidad. Por este motivo se considera que la inmigración en España permitió que pudiera desarrollarse un modelo de crecimiento basado en el desarrollo de sectores de actividad intensivos en factor trabajo y de escasa productividad (Bruquetas y Moreno, 2015: 149). La enorme absorción del mercado laboral de flujos de inmigrantes durante los años de crecimiento tuvo un impacto polarizador, ya que esa tendencia se contrapuso a la de los nativos, que experimentaron un proceso claro de mejora del empleo.

En definitiva, al desagregar por nacionalidad se obtiene una conclusión clara: aunque se generara empleo para todos, si pensamos en términos de la calidad del empleo, vemos que el crecimiento durante la expansión perfiló unos claros ganadores y perdedores.

Sin embargo, la crisis ha afectado más a los nativos ocupados en empleos de baja calidad. Algo lógico, ya que estos datos reproducen cifras absolutas y la población española constituye un grupo mucho más numeroso. Además, el impacto de la crisis sobre el empleo se hizo notar con cierto retraso entre los inmigrantes. En su caso, la caída de empleo masculino de sectores como la construcción se veía compensada en la primera fase de crisis por la creación de puestos de trabajo femeninos en servicios de baja calidad (Arango, 2010: 58; Oliver, 2014).

Aun teniendo en cuenta este desequilibrio, llama la atención la importancia de la contribución de los inmigrantes a la caída de los peores empleos durante la crisis, especialmente en la segunda fase. En realidad, aunque con cierto retraso, no debe sorprender que el impacto global de la crisis haya terminado siendo también muy intenso sobre el colectivo de inmigrantes, ya que la inmigración en España ha tenido un carácter eminentemente laboral (Arango, 2010: 58).

A pesar de las diferencias en cuanto a la intensidad del fenómeno, tanto la evolución de la ocupación de los nativos como la de los inmigrantes han contribuido a generar un proceso de mejora del empleo con la crisis: en ambos casos, la caída de la ocupación se ha concentrado en los empleos de menor calidad. Por tanto, la diferencia fundamental con respecto a la nacionalidad es que mientras que de la larga expansión se beneficiaron fundamentalmente los nativos - quienes se beneficiaron de la creación de empleo de calidad-, la crisis, en términos de ocupación, ha afectado a ambos de forma severa. De ahí que, tras evaluar el ciclo económico completo, se pueda considerar a los inmigrantes como dobles perdedores de un modelo de desarrollo económico.

Además del sexo y la nacionalidad, otra de las características de la oferta de trabajo que se tiene en cuenta habitualmente en estudios sobre fenómenos sociales y económicos es el nivel educativo. Los siguientes gráficos parecen confirmar uno de los supuestos fundamentales de la literatura sobre el cambio tecnológico: que este proceso está ligado a un incremento del nivel educativo 
Gráfico 6. Evolución del $n^{\circ}$ de ocupados (miles) en quintiles, según nivel educativo 1995-2007
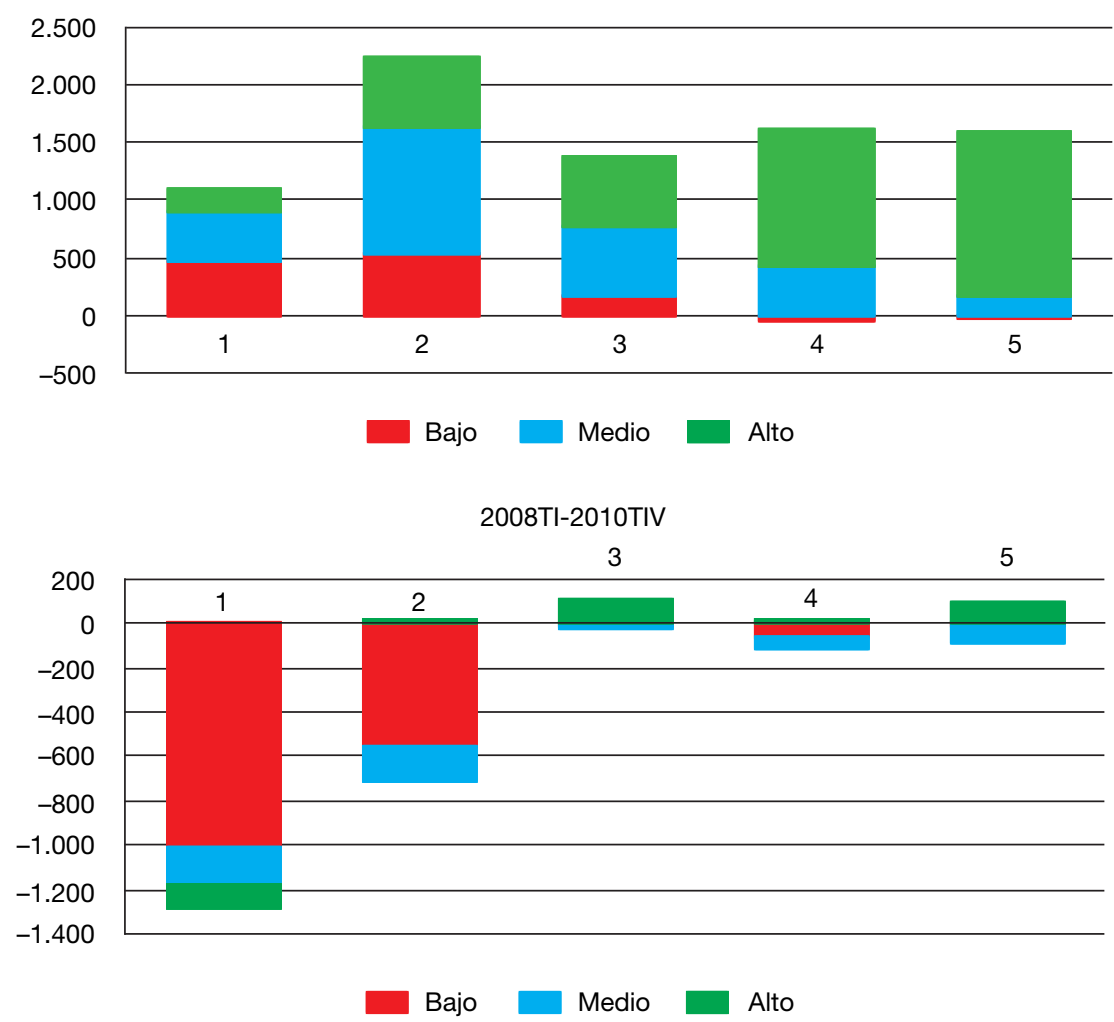

2011TI-2014TII

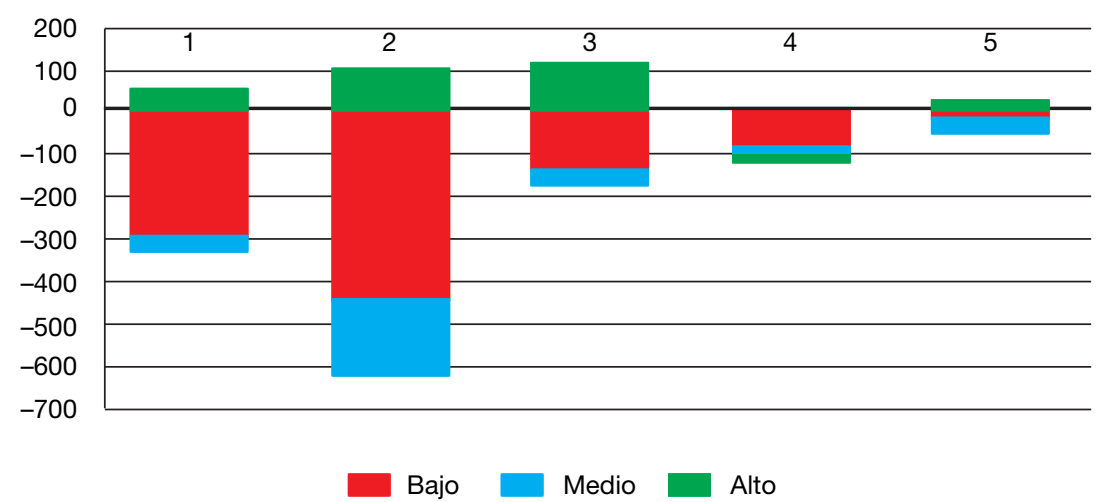

Fuente: elaboración propia a partir de la EU-LFS y la EWCS. 
de la oferta laboral. El gráfico 6a muestra que son los trabajadores que cuentan con un nivel educativo mayor quienes se han beneficiado del aumento de los empleos de calidad, de carácter intelectual y que están relacionados con el uso de las TIC. Si nos fijamos en lo que les ocurre únicamente a estos, vemos que experimentaron un patrón claro de mejora del empleo. Efectivamente, parece que la mejora del logro educativo conduce a un proceso de mejora ocupacional. En cambio, la oferta de trabajo que contaba con un nivel educativo medio y especialmente bajo es la que contribuyó, en mayor medida, al crecimiento de los peores empleos.

Sin embargo, estos datos muestran que siguió habiendo una proporción importante de trabajadores cualificados ocupándose en empleos de calidad media y baja. Gente en muchos casos sobrecualificada para las tareas que desempeñan en sus puestos. Como señalan algunos trabajos, es cierto que en nuestro país la sobrecualificación tiene una incidencia mayor que en el conjunto de países europeos (Cueto et al., 2014: 287; Moreno, 2015), un fenómeno que ya a principios de los noventa se temía que pudiera extenderse (Garrido y Toharia, 1991: 239).

Este desajuste entre las habilidades y las tareas que se llevan a cabo en esos grupos, pone de manifiesto la existencia de un tejido productivo incapaz de absorber la mano de obra cualificada disponible. En cambio, como hemos visto de forma reiterada, en España hasta en la última etapa expansiva el crecimiento de empleos de baja calidad ha sido muy intenso. De esto se deduce que no existe la demanda suficiente para satisfacer la oferta de trabajo cualificado. Las implicaciones de esto en términos prácticos y de cara a pensar en posibles intervenciones públicas son muy importantes, ya que se traducen en que no se puede lograr una mejora del empleo tan solo mejorando el acceso y la calidad de la educación, sino que es necesario impulsar la actividad de sectores de mayor productividad y valor añadido, en los que se desarrollan actividades que se adecúan mejor a las habilidades de esa parte de la oferta de trabajo. Dicho de otro modo, no solo conviene centrarse en tratar de mejorar las características y la empleabilidad de la oferta de trabajo, sino que hay que esforzarse por intervenir por el lado de la demanda, tratando de estimular la creación de un tipo de empleo que satisfaga esa oferta.

Aunque el empleo de calidad intermedia y baja creciera mucho en la época, en términos generales la gente con un nivel educativo bajo se benefició poco de la expansión: su crecimiento fue el más débil y se concentró — casi en el 90\% de los casos- en los dos primeros quintiles. En cambio, la crisis ha sido especialmente dura con ellos $^{8}$, a quienes ha afectado en todos los grupos en general y con mayor intensidad en la parte baja de la estructura. La mejora del empleo experimentada con la crisis ha tenido un coste alto: una enorme

8. Además, quienes tienen un nivel educativo bajo no solo se perfilan como los peor parados a lo largo del ciclo económico anterior, sino que son quienes cuentan con un riesgo potencial mayor de ver sus empleos reemplazados en el futuro debido a la incorporación de nuevas tecnologías (Torrejón, 2017b: 159). 
pérdida de empleo de los menos cualificados. En contraste, los datos sugieren que la educación protege de los efectos de la crisis sobre el empleo: el nivel de ocupación de los más cualificados no solo cayó en menor medida que el del resto, sino que incluso siguió creciendo durante la crisis - aunque en menor medida.

Finalmente, también se ponen en relación estos procesos de cambio con las características de la demanda de trabajo. En particular con la composición sectorial de la estructura productiva, lo que permite tener en cuenta el impacto y la influencia de otros factores - como el institucional- sobre la demanda de empleo. El gráfico 7a resalta la importancia del crecimiento de un sector tan definido como el de la construcción, que hizo una contribución muy importante al crecimiento de los empleos del segundo quintil. Esto refleja la gran dependencia de los ocupados de un sector típicamente cíclico (Banyuls et al., 2009). Algo que ocurrió ya en la segunda mitad de los ochenta (Garrido y Toharia, 1991: 135) y que en la etapa expansiva iniciada a mediados de los noventa se pronunció, y que hubiera tenido que ser un motivo de preocupación de cara al futuro.

Sin embargo, el sector que absorbió más ocupados fue, claramente, el de los servicios. Estos, a diferencia de los primeros, aglutinan actividades muy diversas: desde servicios de baja calidad, como los domésticos y de cuidados, a servicios profesionales y de alto valor añadido, relacionados con el desarrollo de tareas intelectuales, dotadas de autonomía, que requieren el uso de nuevas tecnologías, etcétera. Por este motivo, el crecimiento de este sector contribuyó a engrosar el crecimiento de la ocupación en todos los quintiles — excepto el caso del primero, donde lo hizo en menor medida.

Por tanto, la burbuja inmobiliaria fue un proceso determinante en España en aquella época, ya que impulsó la demanda de empleo de sectores muy definidos — normalmente de baja calidad—, y evitó un reparto más equitativo del mismo. Esto contribuyó de forma decisiva a que se ensanchara la parte baja de la distribución y que el patrón de cambio estructural se sesgara hacia uno con cierta tendencia de carácter polarizador.

Sin embargo, la crisis no se ha centrado sobre los empleos que más crecieron previamente. Los servicios, especialmente en la primera fase, han mostrado una mayor resistencia que la construcción y la industria, ya que las actividades del sector terciario - incluso las de baja calidad, como la hostelería, los cuidados o el servicio doméstico - tienen una mayor capacidad de creación de empleo futuro (Bruquetas y Moreno, 2015: 140). En cambio, los empleos que más han acusado la caída de la demanda son los de baja calidad ubicados en los sectores de la construcción y la industria, cuya evolución ha contribuido de forma decisiva a que hubiera un proceso de mejora ocupacional.

Como resultado, se ve que el efecto de la burbuja inmobiliaria en el empleo fue más nítido tras la crisis. Mientras que en la expansión el crecimiento estuvo más diversificado (debido a que el impacto sobre el empleo de algunos cambios institucionales se complementó con el de otros del lado de la oferta y la demanda de trabajo), la destrucción en la crisis ha estado más focalizada y se ha 
Gráfico 7. Evolución del $n^{\circ}$ de ocupados (miles) en quintiles, según sector
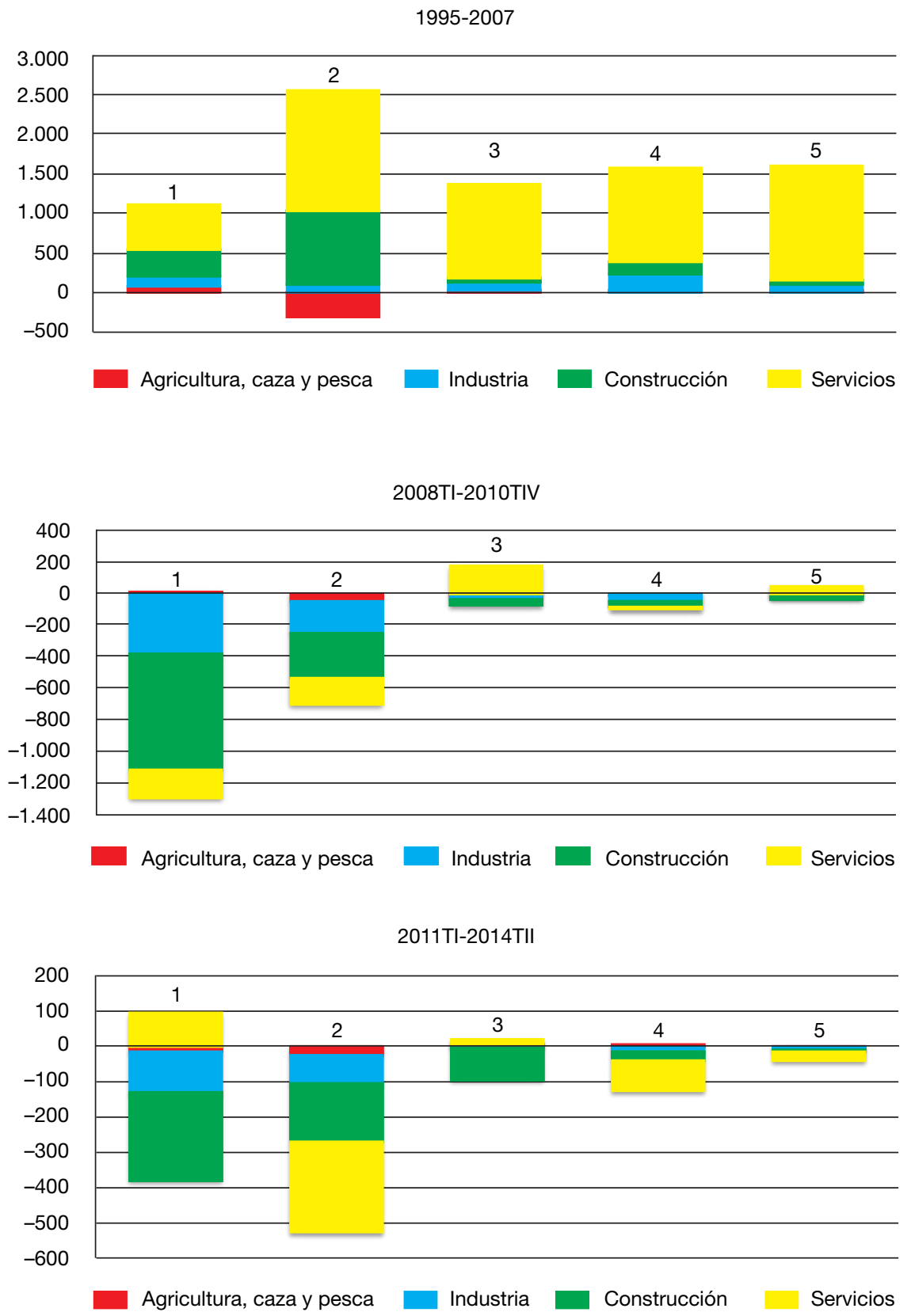

Fuente: elaboración propia a partir de la EU-LFS y la EWCS. 
vinculado de forma más clara a este único cambio. Al desagregar por sectores, se observa algo que se veía con la nacionalidad: la crisis discrimina fundamentalmente según las características y el tipo de empleo. Esto respalda uno de los supuestos básicos de la teoría de segmentación: la importancia de las características materiales del empleo, que lo dota de normas y dinámicas particulares, frente a la perspectiva de que existe un único mercado de trabajo en el que los empleos se distinguen por su grado de calidad o flexibilidad (Peck, 1996: 46-47).

\section{Conclusiones}

Este apartado se divide en dos partes. En la primera, de carácter descriptivo, se detallan los resultados principales. Luego se discute lo que aportan estos datos al debate sobre el impacto social de la crisis.

1. Durante la expansión, fueron los empleos de más calidad y los de calidad intermedia-baja los que más crecieron, lo que permite hablar de una dinámica con cierto carácter polarizador. En cambio, el ajuste de la crisis se ha centrado en los empleos de baja calidad, generando un proceso de mejora ocupacional.

Entre los factores que explican la evolución del empleo en la fase alcista, están la recepción de fuertes flujos de inmigrantes y la disposición de una proporción importante de trabajadores con nivel educativo bajo — dos fenómenos íntimamente relacionados-, que contribuyeron a generar la oferta de trabajo necesaria para satisfacer la demanda de empleos de baja calidad que hubo en la época. Una demanda estimulada, además de por el fuerte crecimiento de servicios no cualificados, por la cantidad de empleo de baja calidad creado en sectores como la construcción. La interacción de estos factores hizo que se dilatara la parte baja de la estructura de empleo, contrarrestando el crecimiento de los empleos de calidad impulsado por el cambio tecnológico, los cambios en el comercio internacional o el aumento del logro educativo de la población nativa.

En cambio, el ajuste de la crisis se ha centrado sobre empleos de baja calidad, muy focalizado en la construcción y la industria. Esto expresa una diferencia fundamental: mientras que los efectos de la expansión discriminaron más por sexo, nacionalidad, educación y otras variables sociodemográficas, la crisis lo hace fundamentalmente por razón del tipo de empleo, afectando intensamente a quienes se ocupan en los sectores en que se centró el ajuste. Es decir, sobre todo a hombres con nivel educativo bajo. En este último caso el efecto de composición es más evidente.

2. El diagnóstico del impacto de la crisis en la estructura de empleo complementa al de quienes han tratado su impacto sobre otras dimensiones del bienestar social, y muestra que la pobreza incide más sobre quienes cuentan con una situación más vulnerable en el mercado laboral (Marí-Klose y Martínez, 2015; Sanzo, 2016), que la bajada de los salarios se ha concentrado en los grupos con sueldos más bajos (Arellano et al., 2013; Bonhomme y Hospido, 2012) e incluso que la percepción subjetiva de bienestar se ha visto más com- 
prometida entre las clases obreras (Marí-Klose et al., 2015). La evidencia de este artículo sobre los efectos de la crisis en distintos tipos de empleos también cuestiona que su impacto haya sido democrático y trasversal, lo que contradice unos supuestos efectos devastadores sobre las clases medias y demuestra que los más perjudicados han sido, de hecho, quienes eran de por sí más vulnerables: los que se ocupaban en los peores empleos.

\section{Referencias bibliográficas}

ACEMOGLu, Daron y Autor, David (2011). «Skills, tasks and technologies: Implications for employment and earnings». En: Ashenfelter, O. y CARD, D. E. (eds.). Handbook of Labor Economics. Amsterdam: Elsevier. <https://doi.org/10.1016/s0169-7218(11)02410-5>

ARANGO, Joaquín (2010). «Después del gran boom: la inmigración en la bisagra del cambio». En: AJA, E.; ARANGO, J. y J. Oliver, J. (eds.). La inmigración en tiempos de crisis. Barcelona: Bellaterra.

ArelLANO, Alfonso; JANSEN, Marcel y JimÉNEZ, Sergi (2013). «Desigualdad salarial, crisis y reformas: bofetadas para todos, pero por turnos». Nada es gratis. Consultado el 12 de enero de 2016. <http://nadaesgratis.es/sergi-jimenez/desigualdad-salarial-crisis-y-reformas-bofetadas-para-todos-pero-por-turnos>.

Arnal, María; Prieto, Carlos y Caprile, María (2012). «Quality of employment: Strategies and interpretations of Spanish employers and trade unions». Revista Internacional de Organizaciones, 9, 45-67.

AUTOR, David (2014). «Skills, education, and the rise of earnings inequality among the 'other 99 percent'». Science, 6186, 843-851. $<$ https://doi.org/10.1126/science.1251868>.

AUTOR, David y DORN, David (2013). «The growth of low-skill service jobs and the polarization of the US labor market». American Economic Review, 5, 1553-1597. <https://doi.org/10.1257/aer.103.5.1553>

AutOR, David; DORN, David y HANSON, Gordon (2013). «Untangling trade and technology: Evidence from local labour markets». The Economic Journal, 584, 621-646. $<$ https://doi.org/10.1111/ecoj.12245>

AutOR, David; KATZ, Laurence F. y KeARNeY, Melissa S. (2006). «The polarization of the US labor market». American Economic Review, 2, 189-194. $<$ https://doi.org/10.1257/000282806777212620>

Autor, David; LeVY, Frank y Murnane, Richard J. (2003). «The skill content of recent technological change: An empirical exploration». The Quarterly Journal of Economics, 4, 1279-1333. <https://doi.org/10.1162/003355303322552801>

Ayala, Luis (2014). Desigualdad y pobreza en España en el largo plazo: la continuidad de un modelo. Madrid: Fundación Foessa. Consultado el 5 de enero de 2016. <http://www.foessa2014.es/informe/uploaded/documentos_trabajo/15102014141318_4885.pdf>.

BANYULS, Josep et al. (2009). "The transformation of the employment system in Spain: Towards a Mediterranean neoliberalism?». En: BosCH, G; LeHNDORFF, S y RUBERY, J. (eds.). European employment models in flux: A comparison of institutional change in nine european countries. Londres: Palgrave Macmillan UK. $<$ https://doi.org/10.1057/9780230237001_10>. 
BEKMAN, Eli; Bound, John y MACHIN, Stephen (1998). «Implications of skill-biased technological change: International evidence». The Quarterly Journal of Economics, 4, 1245-1279. <https://doi.org/10.1162/003355398555892>

BELL, Daniel (1973). The coming of post-industrial society. Nueva York: Basic Books.

BERNARDI, Fabrizio y GARRIDO, Luis (2008). «Is there a new service proletariat? Postindustrial employment growth and social inequality in Spain». European Sociological Review, 3, 299-313. <https://doi.org/10.1093/esr/jcn003>

BonHomme, Stéphane y Hospido, Laura (2012). «The cycle of earnings inequality: Evidence from Spanish social security data». IZA DP 6669. <https://doi.org/10.2139/ssrn.2098547>

BRAVERMAN, Harry (1974). Labor and monopoly capital: The degradation of work in the Twentieth Century. Nueva York: NYU Press.

BRUQueTAS, María y MorenO, Francisco J. (2015). «Precarización y vulnerabilidad de la población inmigrante en la España en crisis. El papel del Estado de bienestar». Panorama Social, 22, 139-151.

CACHÓN, Lorenzo (1997). «Segregación sectorial de los inmigrantes en el mercado de trabajo en España». Cuadernos de Relaciones Laborales, 10, 49-73.

CAIXABANK (2015). Informe mensual 05. Barcelona: Consultado el 12 de febrero de 2016. <http://www.caixabankresearch.com/documents/10180/1416950/ IM+390+Mayo+CAST.pdf/f2b86ac0-4c19-4564-b3ce-e9d6a4c39a43>.

CARABAÑA, Julio (2016). Ricos y pobres: la desigualdad económica en España. Madrid: Catarata.

Carabaña, Julio y SAlido, Olga (2010). «Sobre la difusa relación entre desempleo y pobreza: España en el cambio de siglo». Panorama Social, 12, 15-28.

CASTILlo, J. J. (2010). «Del trabajo, otra vez, a la sociedad: sobre el estudio de todas las formas de trabajo». Sociología del Trabajo, 68, 81-101.

COUNCIL OF ECONOMIC ADVISERS (1996). Job creation and employment opportunities: The United States labor market, 1993-1996. Washington DC: Office of the Chief Economist. <http://babel.hathitrust.org/cgi/pt?id=coo.31924069091811;view=1 up;seq $=7>$.

CES (2012). Memoria sobre la situación socioeconómica y laboral de España en 2011. Consejo Económico y Social. <http://www.ces.es/documents/10180/205054/ MemoriaCES2011.pdf>.

Crouch, Colin (1994). Industrial relations and European state traditions. Oxford: Clarendon Press. <https://doi.org/10.1093/0198279744.001.0001>

Cueto, Begoña; Davia, M. Ángeles; Hernanz, Virginia y Ramos, María (2014). "Trabajo y cualificación». En: García Serrano, C. y Malo, M. Á. (eds.). VII Informe sobre exclusión y desarrollo social en España. Madrid: Fundación Foessa.

DWYER, R. (2013). "The care economy? Gender, economic restructuring, and job polarization in the US labor market». American Sociological Review, 3, 390-416. <https://doi.org/10.1177/0003122413487197>.

ESPING-ANDERSEN, Gøsta (1999). Social foundations of postindustrial economies. Nueva York: Oxford University Press. <https://doi.org/10.1093/0198742002.001.0001>.

Eurofound (2008). More and better jobs: Patterns of employment expansion in Europe. Luxemburgo: Office for Official Publications of the European Communities. <http://www.eurofound.europa.eu/publications/htmlfiles/ef0850.htm>. 
Eurofound (2011). Shifts in the job structure in Europe during the Great Recession. Luxemburgo: Publications Office of the European Union. <http://www.eurofound.europa.eu/publications/htmlfiles/ef1141.htm>.

EuROFOUND (2013). Employment polarisation and job quality in the crisis: European Jobs Monitor 2013. Dublín: Eurofound.

Eurofound (2014). Drivers of recent job polarisation and upgrading in Europe: European Jobs Monitor 2014. Luxemburgo: Publications Office of the European Union.

EUROFOUND (2015). Upgrading or polarisation? Long-term and global shifts in the employment structure: European Jobs Monitor 2015. Luxemburgo: Publications Office of the European Union.

EUROFOUND (2016). What do Europeans do at work? A task-based analysis: European Jobs Monitor 2016. Luxemburgo: Publications Office of the European Union.

FERNÁNDEZ-MACÍAS, Enrique. 2010. "Changes in the structure of employment and job quality in Europe 1995-2007». Madrid: tesis doctoral, Departamento de Sociología y Comunicación, Universidad de Salamanca. <http://gredos.usal.es/ jspui/bitstream/10366/76438/1/DSC_Fernadez_Macias_E_Changes_in_the.pdf>.

FERNÁNDEZ-MACÍAS, Enrique (2012). "Job polarization in Europe? Changes in the employment structure and job quality, 1995-2007». Work and Occupations, 2, 157-182. <https://doi.org/10.1177/0730888411427078>

FERnÁNDEZ-MaCÍAS, Enrique; Bisello, Martina; SARKAR, Sudipa y Torrejón, Sergio (2016). Methodology of the construction of task indices for the European Jobs Monitor. Eurofound. Consultado el 17 de septiembre de 2016. <https://www.eurofound. europa.eu/sites/default/files/ef1617en2.pdf>.

FERnÁNDEZ-MaCíAs, Enrique; Hurley, John y STORRIE, Donald (2012). Transformation of the employment structure in the EU and USA, 1995-2007. Basingstoke: Palgrave Macmillan. <https://doi.org/10.1057/9780230369818>

Fernández-Macías, Enrique; Muñoz de Bustillo, Rafael y Antón, Jose (2014). "Job quality in Europe in the first decade of the 21st Century». MPRA Paper, 58148.

FinA, Lluís (1984). "Cambio ocupacional en España». Información Comercial Española, $607,13-22$.

GaLlie, Duncan (2007). Employment regimes and the quality of work. Oxford: Oxford University Press. <https://doi.org/10.1093/acprof:oso/9780199230105.001.0001>.

Gallie, Duncan; White, Michael; Cheng, Yuan y Tomlinson, Mark (1998). Restructuring the employment relationship. Oxford: Clarendon Press.

García Serrano, Carlos (2011). "Déjà vu? Crisis de empleo y reformas laborales en España». Revista de Economía Aplicada, 56, 149-177.

GARRIDO, Luis (2008a). «Convivencia en pareja, trabajo e inmigración al comenzar el siglo XXI». Economistas, 117, 30-43.

- (2008b). «La inmigración en España». En: GonZÁlez, J. J. y REQuena, M. (eds.). Tres décadas de cambio social en España. Madrid: Alianza Editorial.

- (2010). «El impacto de la crisis sobre la desigualdad en el trabajo». Papeles de Economía Española, 124, 46-68.

GARRIDO, Luis y RODRíGUEZ, Juan Carlos (2011). Estructura ocupacional y carencias formativas en las empresas. Madrid: Forem.

GARRIDO, Luis y TOHARIA, Luis (1991). Prospectiva de las ocupaciones y la formación en la España de los noventa. Madrid: Instituto de Análisis y Estudios Económicos. 
Goos, Maarten y Manning, Alan (2007). «Lousy and lovely jobs: The rising polarization of work in Britain». Review of Economics and Statistics, 1, 118-133. $<$ https://doi.org/10.1162/rest.89.1.118>

Goos, Maarten; Manning, Alan y Salomons, Anna (2009). "Job polarization in Europe». American Economic Review, 2, 58-63. $<$ https://doi.org/10.1257/aer.99.2.58>

- (2010). «Explaining job polarization in Europe: The roles of technology, globalization and institutions». CEP Discussion Paper, 1026, 1-89.

- (2014). «Explaining job polarization: Routine-biased technological change and offshoring». American Economic Review, 8, 2509-2526. <https://doi.org/10.1257/aer.104.8.2509>

Hall, Peter A. y SOSKICE, D. (2001). Varieties of capitalism: The institutional foundations of comparative advantage. Oxford: Oxford University Press.

Marí-Klose, Pau; FernándeZ, Laura y Juliá, Albert (2015). «La percepción subjetiva de la crisis: una aproximación alternativa a procesos de empobrecimiento y amenaza de desclasamiento». Panorama Social, 22, 27-42.

Marí-Klose, Pau y MartíneZ, Álvaro (2015). «Empobrecimiento en tiempos de crisis: vulnerabilidad y (des)protección social en un contexto de adversidad». Panorama Social, 22, 11-26.

MARTín, Antonio y GiBerT, Francesc (2006). «The case of Spain: Growing and secondary labour markets». En: KÖHLER, C.; Junge, K.; SCHRÖDER, T. y STRUCK, O. (eds.). Trends in employment stability and labour market segmentation. Jena: Friedrich-Schiller Universität Jena.

Miguélez, Fausto y PRIETO, Carlos. (2009). «Transformaciones del empleo, flexibilidad y relaciones laborales en Europa». Política y Sociedad, 1-2, 275-287.

MorenO, Almudena (2015). «La empleabilidad de los jóvenes en España. Explicando el elevado desempleo juvenil durante la recesión económica». Revista Internacional de Investigación en Ciencias Sociales, 1, 3-20. $<$ https://doi.org/10.18004/riics.2015.julio.3-20>

Muñoz De Bustillo, Rafael y ANTÓN, José Ignacio (2011). «From the highest employment growth to the deepest fall: Economic crisis and labour inequalities in Spain». En: VAUGHAN-WhiteHEAD, D. (eds.). Work inequalities in the crisis. Evidence from Europe. Cheltenham: Edward Elgar Publishing. <https://doi.org/10.5848/ILO.978-9-221248-86-6_11>

Muñoz de Bustillo, Rafael; FernándeZ-MaCíAs, Enrique; ANTÓN, José I. y EsTEVE, Fernando (2011). Measuring more than money. The social economics of job quality. Cheltenham: Edward Elgar Publishing. <https://doi.org/10.4337/9781849805919>

Muñoz, Jacobo (2014). «¿Qué trabajos ocupan quienes abandonan el desempleo? Diferencias entre españoles y extranjeros en un contexto de cambio económico». Revista Internacional de Sociología, 2, 353-376. <https://doi.org/10.3989/ris.2012.12.18>

OECD (2001). OECD Employment Outlook 2001. París: OECD Publishing. Consultado el 22 de septiembre de 2016. <http://dx.doi.org/10.1787/empl_outlook-2001-en>

OECD (2003). OECD Employment Outlook 2003: Towards more and better jobs. París: OECD Publishing. Consultado el 19 de agosto de 2016. <http://dx.doi.org/10.1787/empl_outlook-2003-en> 
Oesch, Daniel y Rodríguez Menés, Jorge (2011). «Upgrading or polarization? Occupational change in Britain, Germany, Spain and Switzerland, 1990-2008». Socio-Economic Review, 3, 503-531. <https://doi.org/10.1093/ser/mwq029>

Oliver, Josep (2014). «Inmigración y mercado de trabajo en 2013: suave mejora ocupacional y aceleración del envejecimiento poblacional». En: ARANGO, J.; MOYA, D. y Oliver. J. (eds.). Inmigración y emigración: mitos y realidades. Anuario de la Inmigración en España 2013. Barcelona: CIDOB.

PECK, Jamie (1996). Workplace: The social regulation of labor markets. Nueva York: The Guilford Press.

Perez, Carlota (1983). «Structural change and assimilation of new technologies in the economic and social systems». Futures, 5, 357-375. <https://doi.org/10.1016/0016-3287(83)90050-2>

SANZO, Luis (2016). «El impacto social de la crisis de ingresos en España». Zerbitzuan, $62,11-24$. <https://doi.org/10.5569/1134-7147.62.02>

TORREJÓN, Sergio (2016). «La flexibilidad por la vía externa: los factores que impulsan el marcado carácter procíclico del empleo en España». Documentación Social, 178, 55-86.

- (2017a). «Ciclo económico y empleo en España (1995-2014): la dinámica de los flujos y el cambio estructural». Madrid: tesis doctoral, Departamento de Sociología III: Estructura Social y Sociología de la Educación, Universidad Complutense de Madrid.

<https://eprints.ucm.es/49465/>

- (2017b). "Ocupaciones y trabajadores vulnerables: los cambios del lado de la demanda de empleo y sus implicaciones desde el punto de vista de la desigualdad y el género». Revista de Economía Laboral, 14 (2), 137-168. <https://doi.org/10.21114/rel.2017.02.06>.

Wright, Erik O. y DWYER, Rachel (2003). «The patterns of job expansions in the USA: A comparison of the 1960s and 1990s». Socio-Economic Review, 3, 289-325. <https://doi.org/10.1093/soceco/1.3.289>. 


\section{Anexo}

Las siguientes tablas muestran los índices de la propuesta elaborada para el EJM (Fernández-Macías et al., 2016) que he utilizado, así como la puntuación media de cada quintil, información que sirve para caracterizar los grupos de empleos del análisis. He seleccionado los presentes a lo largo de toda la serie, y he omitido algunos de la versión más reciente y completa, que informan acerca de subdimensiones de tareas muy concretas y que considero que no aportan información relevante para este análisis.

Tabla I. Puntuación de los quintiles en los índices de tareas, 1995-2007

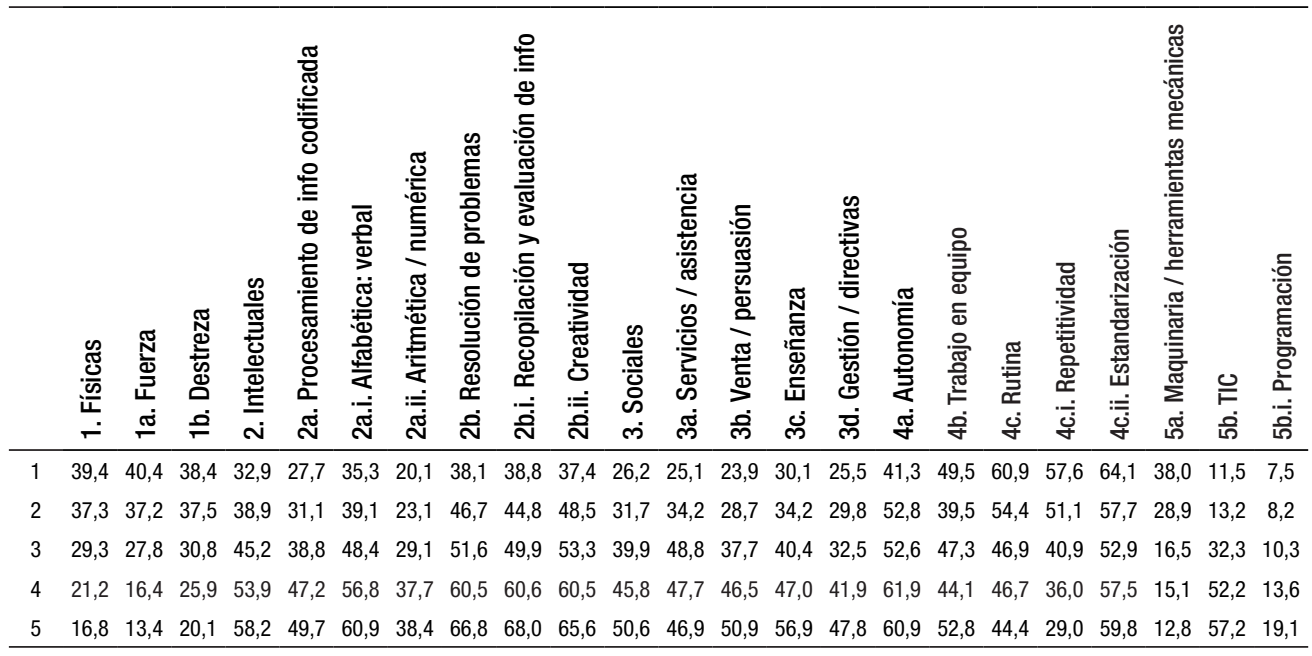

Fuente: elaboración propia a partir de los índices de tareas. 
Tabla II. Puntuación de los quintiles en los índices de tareas, 2008TI-2010TII

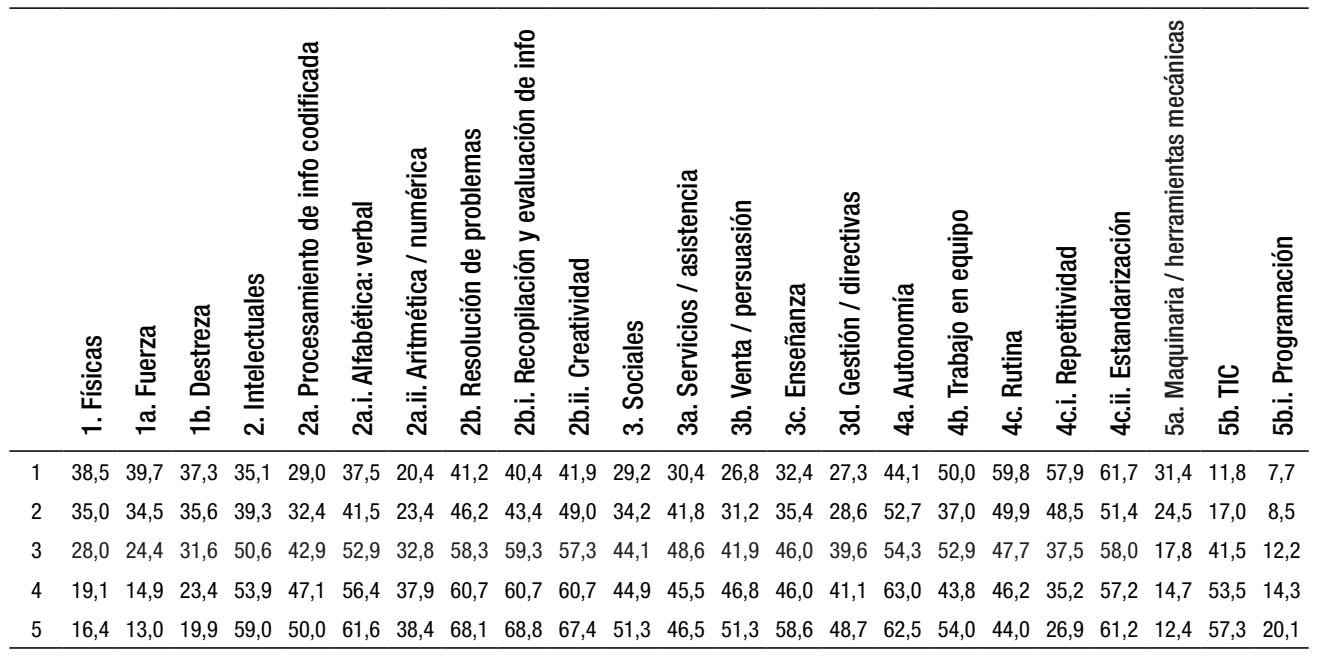

Fuente: elaboración propia a partir de los índices de tareas.

Tabla III. Puntuación de los quintiles en los índices de tareas, 2011TI-2014TII

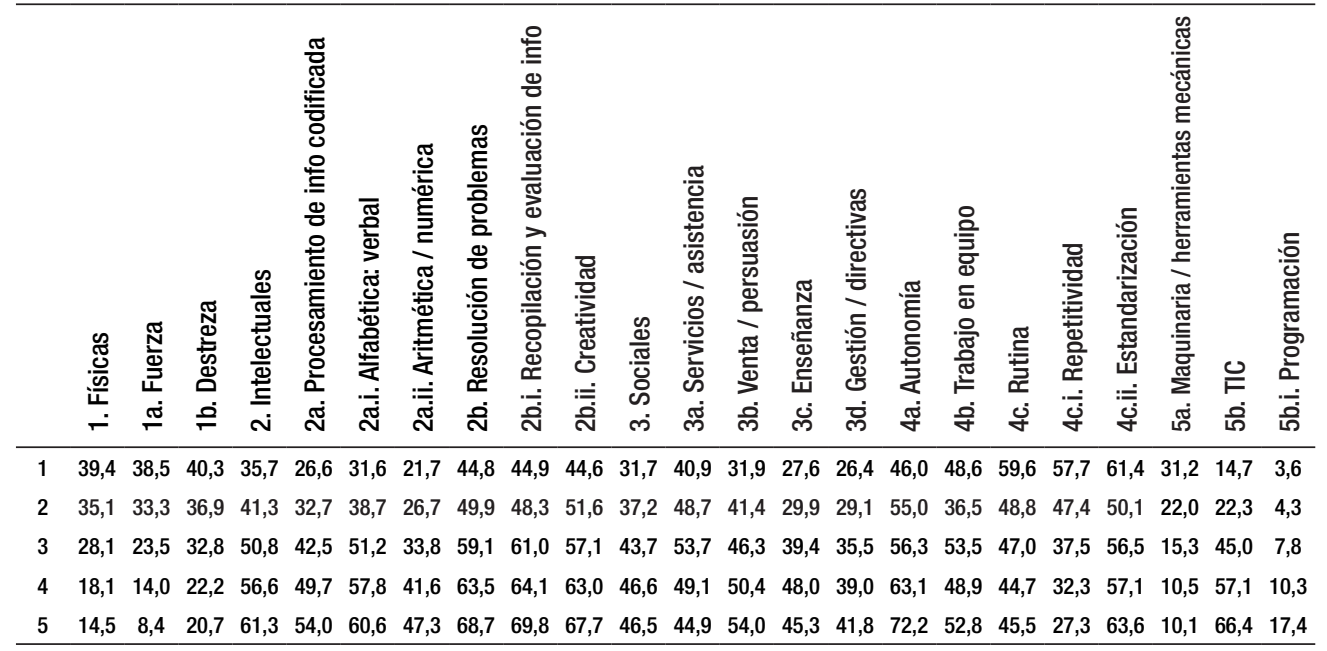

Fuente: elaboración propia a partir de los índices de tareas. 
